\title{
Paradoxical Signaling Pathways in Developing Thymocytes
}

\author{
Aws Alshamsan \\ Nanomedicine Research Unit, Department of Pharmaceutics, College of Pharmacy, King Saud University, Riyadh, Saudi \\ Arabia and National Institute for Nanotechnology, National Research Council, Edmonton, Alberta, Canada.
}

Received, September 23, 2011; Revised, October 5, 2011; Accepted, October 7, 2011; Published, October 7, 2011.

Table of Contents

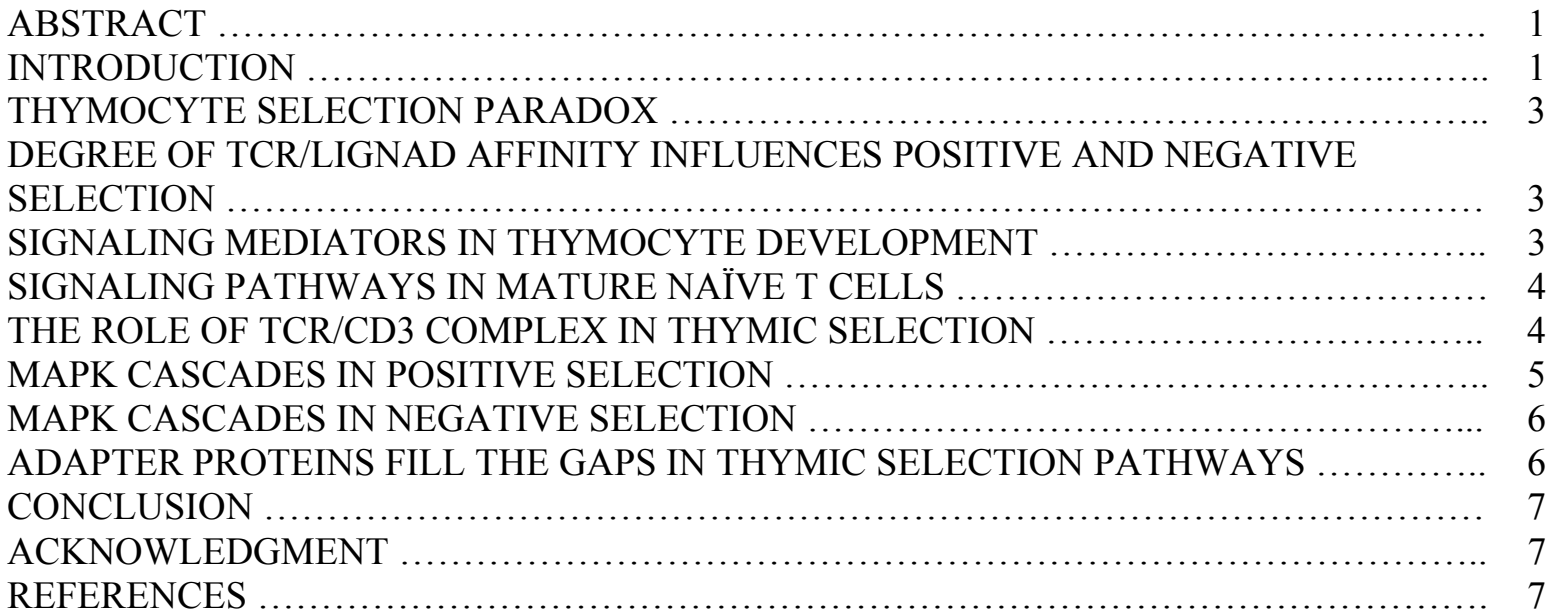

\begin{abstract}
Thymocytes are subjected to processes of selection during their life in the thymus; negative selection for autoreactive thymocytes and positive selection for self-MHC restricted self-tolerant cells. Interestingly, signals for positive or negative selection originate from the same receptor. More importantly, evidence showed that both death and survival signals are mediated by the MAPK pathway. The degree and order of ERK activation, but not other MAPK proteins, has been found to be different in either cases of cell fate. Therefore, it is suspected that the kinetics of ERK after activation may dictate cell death or survival. There are two important GEF proteins that are involved in Ras/ERK activation, RasGRP and SOS. It is thought that the level, order and kinetics of ERK are influenced upstream by the type of GEF. This review discusses the role of both GEF proteins in positive and negative selection and how this reflects on ERK activation.
\end{abstract}

This article is open POST-PUBLICATION REVIEW. Registered readers (see "For Readers") may comment by clicking on ABSTRACT on the issue's contents page.

\section{INTRODUCTION}

The thymus is a primary lymphoid organ that is responsible for development and maturation of precursor hematopoietic cells into mature $\mathrm{T}$ cells. Thymic environment plays the major role in $\mathrm{T}$ lymphocyte development. However, the mechanism(s) by which this development is regulated is still debatable. Arising from hematopoietic stem cells, T cell progenitors migrate from the bone marrow to the thymus where they reside and take the name thymocytes. These thymocytes accept one of two fates: either differentiation or death depending on the signals originated from the $\mathrm{T}$ cell receptor (TCR) and generated by thymic microenvironment (1). These signals include: (i) cells of hematopoietic origin such as antigen presenting cells (APCs) and (ii) non-hematopoietic origin such as various types of

Corresponding Author: Dr. Aws Alshamsan, P. O. Box 2457, Riyadh 11451, Saudi Arabia. Email: aalshamsan@ksu.edu.sa 
thymic epithelial cells (TEC) and mesenchymal fibroblasts (2). Thymocytes pass through different morphological stages characterized by their surface markers. Upon entering the thymus, they lack the expression of both CD4 and CD8, also known as double negative (DN). In the thymic cortex, DN cells progress through four developmental stages distinguished by the expression of CD44 and CD25: $\mathrm{DN} 1\left(\mathrm{CD} 44^{+} / \mathrm{CD} 25^{-}\right), \mathrm{DN} 2\left(\mathrm{CD} 44^{+} / \mathrm{CD} 25^{+}\right), \mathrm{DN} 3$ $\left(\mathrm{CD} 44^{-} / \mathrm{CD} 25^{+}\right)$, and DN4 (CD44/CD25 ). In DN3 stage, rearrangement of TCR $\gamma, \delta$ and $\beta$ loci is evident and the two lineage of T cells $\alpha / \beta$ and $\gamma / \delta$ diverge (2). The assembly of a functional $\beta /$ preTCR $\alpha$ complex leads to an $\alpha / \beta$ committed lineage which involves the expression of CD4 and CD8 coreceptors and rearrangement of the $\alpha$ locus. At this stage of thymocytes development, also known as double positive (DP), thymocytes are capable of expressing $\alpha / \beta$ heterodimeric TCR at the cell surface (2). This enormous repertoire of DP immature thymocytes will be subjected to positive and negative selections. According to proper TCRmediated interaction with thymic stroma, only cells with functional TCR that are not autoreactive will be allowed to pass these checkpoints, while thymocytes with non-reactive TCR will die by neglect (3). A small fraction of thymocytes (less than 5\%) will make it to this stage and will downregulate the expression of either CD4 or CD8 coreceptors to be single positive (SP) $\mathrm{T}$ cells and committed to either $\mathrm{CD}^{+}$(helper) or $\mathrm{CD}^{+}$ (cytotoxic) lineages and ready to leave the thymus as mature naïve T lymphocytes (2-4). The different stages of thymocyte development are shown in (Figure 1).
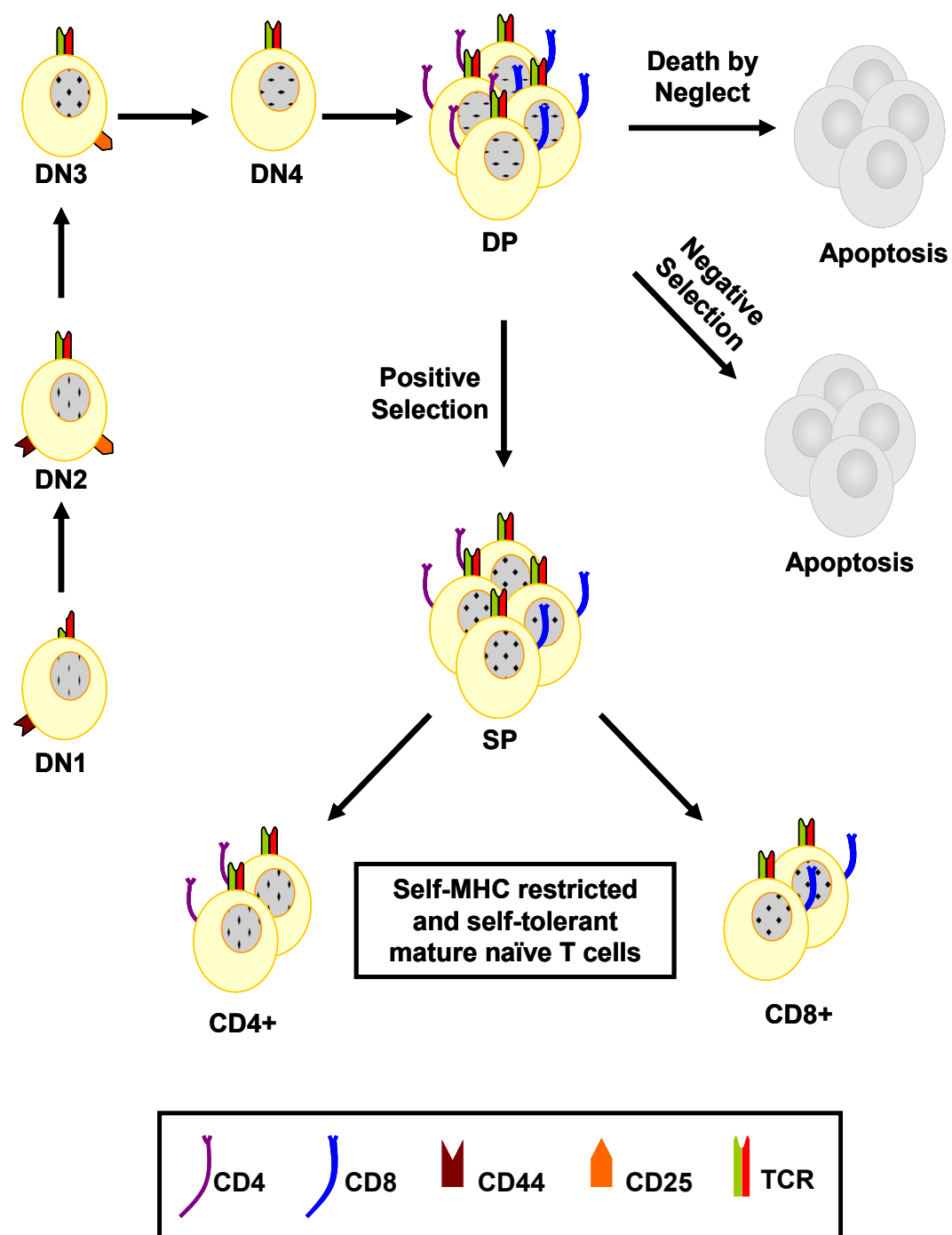

Figure 1. Depiction of thymocytes development into naïve-mature T cells through T cell receptor (TCR) activation. 
Due to the complexity of TCR interaction with antigens of different affinities, the relationships among all variables involved in this process was not fully understood. Faro et al. have developed a quantitative model for thymic selection that provided estimates of the fractions of thymocytes going through the events described above (3). During $\mathrm{T}$ cell life, a molecular signal arises from TCR triggering by peptide presented on the context of the major histocompatibility complex (peptide:MHC). This signal controls various stages of $\mathrm{T}$ cells development, proliferation, differentiation or even their death $(5,6)$. TCR is a unique type of receptors because, upon stimulation, it delivers signals that could lead to one of two opposing fates i.e. survival or death (1). This paradox has been connected to different molecular signaling events in either case. This review will explain the different signaling pathways involved in positive and negative selections and elaborate on relative importance and mutual exclusiveness in both scenarios.

\section{THYMOCYTE SELECTION PARADOX}

Thymus microenvironment provides a testing panel for the huge repertoire of DP thymocytes. According to its specificity, TCR engagement of self-reactive DP cells will trigger an apoptotic signal resulting in negative selection of these cells. On the other hand, TCR engagement can also trigger a survival signal and further differentiation of non-self-reactive DP cells i.e. positive selection. Immunologists have studied this paradox for decades (7). Still there is no definite scenario that explains these phenomena. Nevertheless, it had been accepted that both positive and negative selections depend on the type of peptide presented to the thymocyte $(2,7-9)$, which reflects its TCR affinity to (peptide:MHC) complex $(1,3)$ and accordingly, dictates the kinetics pattern of the signaling molecules, and hence cell fate $(1,2,10$, 11).

\section{DEGREE OF TCR/LIGNAD AFFINITY INFLUENCES POSITIVE AND NEGATIVE SELECTION}

It is generally accepted that positive-selection is induced by low affinity ligands, while high affinity ones will induce negative selection (1-4). Several explanations were addressed to how a thymocyte discriminates between (peptide:MHC) ligands of different affinities. One explanation was based on the ability of ligands to occupy TCRs i.e. avidity, where high-affinity ligands would occupy more TCRs on the cell surface and the developing thymocyte will simply count the numbers of ligated receptors $(1,4)$. This model is not proved experimentally yet (4). Alternatively, another explanation took into account the ligand occupancy time. It suggests that, upon TCR engagement, an early signal is triggered leading to positive selection followed by a late signal that leads to negative selection. Low-affinity ligands would have short a occupancy time that is confined to early signal induction and hence positive selection, while highaffinity ligands have longer occupancy time which would initiate both early and late signal and hence induction of negative selection; an idea known as kinetic proofreading $(1,4,12,13)$. Nevertheless, the previous explanations did not consider the coreceptors CD4 and CD8, which have an influence on the strength of TCR engagement by binding to MHC-II and MHC-I, respectively. TCRs that have coengaged CD4 are thought to deliver a stronger signal because Lck is bound to CD4 more tightly than it is to CD8 (1). Consistently, it was observed that in the presence of the dominant-negative form of Lck, thymocytes expressing a class II MHCrestricted TCR develop into $\mathrm{CD} 8 \mathrm{~T}$ cells, while those which express a class I MHC-restricted TCR will develop into CD4 $\mathrm{T}$ cells in the presence of the constitutively active form of Lck $(1,14)$. Moreover, Lui and Bosselut proved that the duration of TCR engagement controls the differentiation of DP thymocytes into CD4 or CD8 SP cells (15). Together, these findings indicate that a thymocyte TCR is capable for distinguishing ligands of different affinities and, accordingly, initiate either a survival or death signals in the DP cells.

\section{SIGNALING MEDIATORS IN THYMOCYTE DEVELOPMENT}

More studies have shed light on the molecular mechanisms by which positive or negative selection ensues. It was accepted early on that thymic selection processes are mediated by MAPK pathway $(1,2,4)$. To address this proposition, studies have focused on the TCR/CD3 complex $(\alpha$, $\beta$ and CD3 chains), adapters and enzymatic proteins involved in MAPK pathway in the context of developing thymocytes. 


\section{SIGNALING PATHWAYS IN MATURE NAÏVE T CELLS}

Upon TCR engagement with its ligand, (peptide:MHC-I) in case of $\mathrm{CD}^{+}$and (peptide:MHC-II) in case of $\mathrm{CD}^{+} \mathrm{T}$ cells, a network of several enzymes and adapter proteins ensue, allowing the $\mathrm{T}$ cell to respond i.e. proliferation and differentiation.

The coreceptors (CD4 or CD8) bring Src family kinases (Lck and Fyn) in close proximity to the TCR complex, where they become phosphorylated. In turn, they phosphorylate immunoreceptor tyrosine-based activation motifs (ITAMs) located on the cytoplasmic tail of the CD3 complex. ITAMs phosphorylation provides docking sites for Syk family proteins, especially the zeta-associated protein of $70 \mathrm{kDa}$ (ZAP-70). This catalyzes many initial phosphorylation reactions including the phosphorylation of two adapter proteins: linker for activation of $\mathrm{T}$ cells (LAT) and SH2-domain containing leukocyte protein of $76 \mathrm{kDa}$ (SLP-76) $(16,17)$. LAT contains nine tyrosine residues eligible for phosphorylaion and to which $\mathrm{SH} 2-$ domain containing proteins bind $(1,2)$; mainly: phospholipase C $\gamma$ (PLC- $\gamma$ ), p85 subunit of phosphoinositol-3 kinase (PI3K), the adapter proteins Grb2 and its homologue GADS $(17,18)$. The degree of LAT phosphorylation is thought to be highly associated with driving cell response to positive or negative selection $(1,4)$.

Phosphatidyl-inositol-4, 5 bisphosphate (PIP2) is a target for class I PI3K which phosphorylate PIP2 to become phosphatidyl-inositol-3, 4, 5 trisphosphate (PIP3), an essential signaling molecule during the sustained response to lymphocyte activation $(19,20)$.

PIP2 is also hydrolyzed by PLC- $\gamma$ generating inositol-1, 4, 5 trisphosphate (IP3) and diacylglycerol (DAG). IP3 enhances the release of $\mathrm{Ca}^{2+}$ ions from the endoplasmic reticulum to the cytosole that act in a part to activate the $\mathrm{Ca}^{2+} /$ Calmodulin/Calcineurin pathway. This in turn activates the transacription factor nuclear factor of activated T cells (NFAT) $(17,18)$. Furthermore, $\mathrm{Ca}^{2+}$ ions act with DAG to activate protein kinase $\mathrm{C}$ (PKC). The latter activates the transcription factor NFkB. DAG activates RasGRP as well, a guaninenucleotide exchange factor (GEF) that is upstream in mitogen-activated protein kinase (MAPK) pathway.
Grb2 binds to a phosphorylated spot on LAT, and then binds to the GEF protein, the Son of Sevenless (SOS); which, like RasGRP, activates MAPK pathway. RasGRP or LAT-Grb2-SOS directed activation of Ras will activate one or more MAPK, namely extracellular signal-regulated kinase (Erk), c-Jun NH2-terminal kinase (JNK) and/or p38 $(2,10,11,21)$. These phosphorylate the transactivator Elk-1 that results in the formation and phosphorylation of another transactivator AP-1 (17). MAPK pathway is suggested to be the instrument for thymocyte development (21).

The SH3 domain of GADS constitutively associates with the proline-rich region of SLP-76 and comes in close proximity to ZAP-70, hence it is phosphorylated. Phosphorylation of the N-terminus of SLP-76 provides docking sites for Vav, the adapter Nck and the Tec kinase Itk. Another proline-rich region of SLP-76 binds to an SH3 domain of LAT-bound PLC- $\gamma$. This interaction is believed to serve the localization of PLC- $\gamma$ and bringing it closer to Itk, which is an activator for PLC- $\gamma(2,17)$.

\section{THE ROLE OF TCR/CD3 COMPLEX IN THYMIC SELECTION}

Bäckström and colleagues studied the role of TCR $\alpha$-chain-connecting peptide domain $(\alpha-\mathrm{CPM})$ in positive selection (22). By the use of transgenic mice lacking $\alpha-C P M$ in their TCR, they found that thymocytes were blocked in positive selection but could undergo negative selection normally. Later on, Werlen et al. (10), using transgenic mice with mutant $\alpha$-CPM TCR, examined the contribution of MAPK in thymic selection. They showed that thymocytes expressing mutant $\alpha$-CPM TCR failed to activate ERK specifically after stimulation with a positively selecting peptide, while the other MAPK, JNK and p38, were normally activated. On the other hand, negative selection was not overwhelmed after stimulation with a negatively selecting peptide. Important findings were also concluded from this work. They noticed that, in wild type mice, the kinetics of MAPK activation is different in positive and negative selection. In positive selection, ERK is activated in slow onset but sustained manner compared to negative selection. Moreover, they noticed that mutant $\alpha$-CPM TCE failed to coimmunoprecipitate $\mathrm{CD} 3 \delta$. Taken together, they proposed that a low-avidity ligand uses $\alpha-C P M$ and 
$\mathrm{CD} 3 \delta$ to promote recruitment of activated signaling mediators including Lck, ZAP-70 and LAT.

It was observed that $\mathrm{CD} 3 \delta$ DP cells fail to undergo positive selection. This is also the case in mice expressing inactive components of MAPK pathway (2). Delgado and colleagues (11) studied MAPK pathway induction in CD3 $\delta$ thymocytes upon TCR engagement. A profound loss in ERK, but not other MAPK, activation was observed in $\mathrm{CD} 3 \delta^{-/-}$thymocytes. This inactivation was not observed in $\mathrm{CD} 3 \delta$ or thymocytes lacking $\mathrm{CD} 3 \delta$ cytoplasmic tail, indicating that requirement of the CD3 $\delta$ for positive selection is not in the cytoplasmic tail. Furthermore, the defect correlates with severely impaired tyrosine phosphorylation of LAT and $\mathrm{CD} 3 \zeta$ chain that is localized to membrane lipid raft upon TCR engagement. Surprisingly, other data showed that the overall process of positive selection could occur in the absence of CD3 $\zeta$ chain ITAMs (2). Recent studies suggest that CD8 is linked to the TCR by way of CD3 $\delta$ and/or $\alpha$-CPM and hence stabilize TCR interaction with its ligand (1). Interestingly, some data suggest that CD8-deficient cells could undergo negative but not positive selection (4). It could be concluded that $\mathrm{CD} 3 \delta$ and $\alpha-C P M$ might have no role in negative selection because high affinity ligands, involved in negative selection, are sufficient themselves to engage thymocytes TCR without the aid of CD8 coreceptor.

To address the role of CD3 ITAMs, Haks et al. (21) created knock-out mice in which the CD3 $\gamma$ chain is replaced with a mutant signaling-deficient one. They noticed that the mutation resulted in considerable impairment in positive selection. Moreover, LAT and ERK phosphorylation was profoundly impaired but not that of JNK. Nevertheless, the binding and dissociation constants of agonist ligands were not affected by this mutation.

\section{MAPK CASCADES IN POSITIVE SELECTION}

Studies on MAPK cascades in thymocytes indicated that no single MAPK is exclusively associated with positive or negative selection $(1,4)$. It was thought that distinct signals drive DP thymocytes toward positive or negative selection, where ERK is responsible for positive selection and JNK and p38 for negative selection (21). Studies of PC 12 pheochromocytoma cells demonstrate that cell survival and death are controlled by opposing actions of ERK and JNK-p38 pathways, which could be applied on the thymic selection process (23). These thoughts were confronted when Mariathasan and colleagues (24) provided biochemical evidence of ERK activation in response to a negatively selecting peptide. It was shown that positively selecting ligands induced weaker activation of ERK consistently with the affinity/avidity model discussed above. Interestingly, they were capable to switch cell fate from being negatively selected to being positively selected by the addition of MEK inhibitor with a negatively selecting ligand. These data demonstrated that the degree of ERK activation influences cell fate $(1,24)$. Furthermore, the inhibition of upstream molecules in TCR activation cascades diminish both positive and negative selections (25).

Treatment of Jurkat cells with butyric acid, known to induce cytotoxicity and apoptosis, decreased ERK but not JNK or p38 phosphorylation (26). DNA microarrays and Northern blots analyses of unstimulated Jurkat cells revealed a repression in the expression of Recombination Activation Gene-1 and 2 (RAG-1 and RAG-2) upon low level and sustained activation of ERK (27). This implies the physiological importance of ERK activation as a reflection of correct TCR recombination. Moreover, some studies showed that Ras activation by TCR engagement inhibits glucocorticoid-induced apoptosis (28).

More studies have also shown that ERK is induced slowly and over a sustained period of time by low-affinity ligands, whereas high affinity ligands induce an intense and transient burst or ERK activity $(2,4,29)$. It is also possible that the order of activation of the various MAPK pathways determines which transcription factors are triggered and this dictates cell fate $(2,4)$. In a very recent study, Costello et al. (30) studied the molecular mediators downstream of ERK activation during thymocyte development. They have characterized the function of SAP-1 (Elk4) using SAP-1deficiend mice. They demonstrated a profound defect in positive but not negative selection although ERK activation was normal. Moreover, the activation of Erg-1, a zinc-finger Elk-induced transcription factor, was dramatically impaired. This suggests the importance of Erg-1 in positive but not negative selection $(2,30)$. 
Ras, upstream in the MAPK pathway, is activated in a positive manner by at least two GEF proteins namely RasGRP and SOS. The latter is recruited to the TCR activation complex by interacting with the adapter proteins Grb2 and LAT. Therefore, it was assumed that the Grb2/SOS pathway is the mechanism by which Ras is activated and positive selection occurs. Yet, there was a missing link between Ras/ERK activation and how this activation is dependent on PLC- $\gamma$ (31). This was with the discovery of RasGRP, a DAGregulated GEF, which is now accepted to be responsible for ERK activation in positive selection (32). Interestingly, recent data revealed a connection between calcineurin B1 regulatory subunit and positive selection. This gives more significance for PLC- $\gamma$, but more studies have to be completed in that aspect (33). Using the offspring of RasGRP1 transgenic mice bred to RAG- $2^{-/-}$mice, RasGRP induction of DN cell maturation and enhancement of positive selection were demonstrated (34). Furthermore, Priatel et al. (35) produced two lines of RasGRP1 ${ }^{-1-}$ TCR transgenic mice with weak or strong affinity to the selecting ligand. They showed that a weakly selecting TCR is crucially dependent on RasGRP1 for its positive selection. However, ERK activation and positive selection of thymocytes expressing a strongly selecting TCR are much less dependent on RasGRP1. Notably, RasGRP1 deficiency had no effect on JNK-p38 pathways or negative selection (35).

\section{MAPK CASCADES IN NEGATIVE SELECTION}

Accumulation of data suggests the connection of MAPK activation in negative selection to Grb2/SOS pathway $(1,2,4)$. Gong et al. (36), using $\mathrm{Grb}^{+/-}$mice, showed a decrease in JNK and p38 expression but not ERK. Furthermore, they demonstrated impairment to negative but not positive selection in $\mathrm{Grb}^{+/-}$thymocytes $(35,36)$. More studies have shown that both positively and negatively selecting ligands induce JNK and p38 with similar kinetics (10). Only ERK is induced in different kinetics and order by positively or negatively selecting ligands $(1,2,4)$. Negatively selecting peptides induce fast, intense and transient burst of ERK activity, which is before JNK and p38 in negative selection $(1,2,29)$. Studies have been done to address the JNK role in negative selection.
It has been shown that thymocytes expressing the dominant negative JNK transgene were less likely to undergo negative selection while positive selection was not impaired $(37,38)$. Another study addressed the role of $\mathrm{p} 38$. Experiments done in fetal thymic organ culture (FTOC) showed impaired negative but not positive selection in the presence of a pharmacological inhibitor of p38 (39). Other mechanisms do exist for negative selection e.g. Fas/Caspases pathway. However, the discussion of these mechanisms is beyond the scope of this review $(2,4)$.

\section{ADAPTER PROTEINS FILL THE GAPS IN THYMIC SELECTION PATHWAYS}

Studies on adapter proteins explicate their role in localizing and interacting with effector signaling mediators. Along with Grb2, three other adapters are thought to be indispensable in TCR signaling: LAT, SLP-76 and GADS (17).

GADS is a hematopoietic-lineage-specific Grb2 family member protein. It serves as a connection between LAT and SLP-76 $(1,17)$. Two groups have studied the role of GADS in developing thymocytes. Kikuchi et al. generated transgenic mice expressing an $\mathrm{SH}$-deleted dominant-negative form of Gads (40). They noticed a profound reduction in the total number of thymocytes. Furthermore, the DN3 thymocyte population was significantly increased. Interestingly, after stimulation with anti-CD3 $\mathrm{mAb}$, the degree of phosphorylation of SLP-76, ZAP-70 and ERK was dramatically decreased in mutant thymocytes compared to the wild type. Yoder and colleagues generated a Gads-deficient mouse by gene targeting. They noticed a severe block in DN thymocyte proliferation. They also showed that positive and negative selections were impaired in vivo upon stimulation of CD3. Consistent with the previous finding, immunoprecipitation experiments revealed that the association between SLP-76 and LAT was uncoupled in GADS-deficient thymocytes (41). It could be inferred that associating SLP-76 to LAT is a must for TCR signaling.

Despite the recognized role of LAT and SLP-76 in promoting signaling in mature $\mathrm{T}$ cells, very little is known regarding their contribution in the outcome of thymic selection. This is due mainly to a lack of experimental systems where the expression or function of LAT or SLP-76 is manipulated specifically at the DP stage of 
thymocyte development (42). SLP-76 is a target of ZAP-70 phosphorylation, which is negatively regulated by hyper-phosphorylation of ZAP-70 (43). Kumar et al. have demonstrated a differential role of SLP-76 domains in thymocyte development (44). In their study, SLP-/- mice where reconstituted with SLP-76 deleted mutant transgenes. They found that the N-terminal domain deletion failed completely to restore thymocyte development. This is probably because it is a site for Itk attachment. Itk is an important activator for PLC $-\gamma$ which leads to the production of DAG and hence RasGRP activation (17). It is also a site for Vav1 attachment, which is a GEF protein transduces signals via PLC- $\gamma$ which eventually activate RasGRP and it is required for SOS recruitment to $\operatorname{LAT}(45,46)$. Decrease thymic population and impaired transition from DP to SP stage were observed in mice reconstituted with Gads-binding site and SH2-deleted domains. ERK activation was decreased in Gads-binding deleted domain but almost normal in SH2-deleted domain mice (44). Taken together with the previous findings, these data supports the importance of GADS/LAT/SLP-76 complex for apt TCR signaling $(1,2,17,42)$.

LAT has nine tyrosine residues, and differential phosphorylation of these tyrosine side chains might control the speed of signaling (4). It is conceivable that positive selection induces a delay in phosphorylation of LAT, involving Tyr175 and Tyr195, thus leading to the formation of an unstable complex of LAT/GADS/SLP-76/PLC- $\gamma$. This may cause weak activation of the latter kinase and eventually slow and sustained ERK activation (1). On the other hand, high affinity ligands rapidly phosphorylate these tyrosine residues in addition to Tyr136, which ensures a quick and strong activation of PLC- $\gamma$ (1). Interestingly, Grb2 was shown to be recruited to additional tyrosine phosphorylation (Tyr113, Tyr132 and Tyr235) (4).

\section{CONCLUSION}

The capability of developing-thymocyte TCR to distinguish two opposing cell fates in response to its stimulus has always been a fascinating phenomenon. Many models were proposed to explain this phenomenon. It is not easy to point out a particular subcellular molecule. Albeit, a collective view of these models leads to the conclusion that the order and kinetics of the MAPK cascades determine the ultimate fate of a developing thymocyte. In physiological conditions, positively selecting ligands induce slow, late and sustained activation of ERK, which is mediated by RasGRP activation of Ras. On the other hand, negatively selecting ligands induce a quick, early and transient activation of ERK mediated by the Grb2/SOS pathway. The gist of these two different pathways is the different kinetics in ERK production, since it was shown that changing ERK kinetics switched cell fate from negative to positive selection. Although studies did not address this issue, RasGRP and Grb2/SOS pathways do not seem to be mutually exclusive because their activation depends on the degree of phosphorylation of the proteins involved in upstream events e.g. extensive phosphorylation of LAT activates Grb2/SOS pathway leading to negative selection. Yet, the inhibition of MEK downstream Grb2/SOS pathway has lead to positive selection. It is not obvious whether this switching to positive selection is a direct effect of Grb2/SOS pathway inhibition or an indirect stimulation of RasGRP. More importantly, only a few studies discussed signaling events after ERK activation with different kinetic patterns. It was suspected that different transcription factors are generated by different levels of ERK activation or the kinetic profile might trigger regulatory proteins production that influences cell fate $(47,48)$. Nevertheless, from the experimental evidences shown, it is conceivable to say that RasGRP mediates ERK activation in positive selection in a specific kinetic profile and Grb2/SOS mediated ERK activation in a different kinetic pattern for negative selection.

\section{ACKNOWLEDGMENT}

The author is grateful to the support of the College of Pharmacy Research Center and the Deanship of Scientific Research, King Saud University, Riyadh, Saudi Arabia.

\section{REFERENCES}

1. Werlen, G., et al., Signaling life and death in thymus: timing is everything. Science, 2003. 299: p. 1859-63.

2. Starr, T.K., S.C. Jameson, and K.A. Hogquist, Positive and Negative Selection of T Cells. Annu. Rev. Immunol., 2003. 21: p. 139-76.

3. Faro, J., et al., The impact of thymic antigen diversity on the size of the selected $\mathrm{T}$ cell repertoire. J. Immunol., 2004. 172(4): p. 2067-75. 
4. Palmer, E., Negative selection-clearing out the bad apples from the T-cell repertoire. Nat. Rev. Immunol., 2003. 3(5): p. 383-91.

5. Dorfman, J.R. and R.N. Germain, MHC-dependent survival of naive T cells? A complicated answer to a simple question. Microbes and Infection, 2002. 4: p. 547-54.

6. Tan, S.-L. and P.J. Parker, Emerging and diverse roles of protein kinase $\mathrm{C}$ in immune cell signalling. Biochem. J., 2003. 376(3): p. 545-52.

7. Anderson, M.S., et al., Projection of Immunological Self Shadow Within the Thymus by the Aire Protein. Science, 2002. 298: p. 1395-1401.

8. Derbinski, J., et al., Promiscuous gene expression in medullary thymic epithelial cells mirrors the peripheral self. Nat. Immunol., 2001. 2(11): p. 103239.

9. Ohashi, P.S., Exposing Thy Self. Science, 2002. 298: p. 1348-49.

10. Werlen, G., B. Hausmann, and E. Palmer, A motif in the ab T-cell receptor controls positive selection by modulating ERK activity. Nature, 2000. 406: p. 42226.

11. Delgado, P., et al., CD3d couples T-cell receptor signalling to ERK activation and thymocyte positive selection. Nature, 2000. 406: p. 426-30.

12. McKeithan, T.W., Kinetic proofreading in T-cell receptor signal transduction. Proc Natl Acad Sci USA, 1995. 92(11): p. 5042-6.

13. Hlavacek, W.S., et al., Kinetic proofreading in receptor-mediated transduction of cellular signals: receptor aggregation, partially activated receptors, and cytosolic messengers. Bull Math Biol, 2002. 64(5): p. 887-911.

14. Hernandez-Hoyos, G., et al., Lck activity controls CD4/CD8 $\mathrm{T}$ cell lineage commitment. Immunity, 2000. 12(3): p. 313-22.

15. Lui, X. and R. Bosselut, Duration of TCR signaling controls CD4-CD8 lineage differentiation in vivo. Nat. Immunol., 2004. 5(3): p. 280-288.

16. Alonso, A., et al., Tyrosine phosphorylation of VHR phosphatase by ZAP-70. Nat. Immunol., 2003. 4(1): p. 44-48.

17. Jordan, M.S., A.L. Singer, and G.A. Koretzky, Adaptors as Central Mediatore of Signal Transduction in Immune Cells. Nat. Immunol., 2003. 4(2): p. 110-116.

18. Hardy, R.R., et al., Signaling Through Imune System Receptors, in Immunobiology: the immune system in health and disease, C.A. Janeway, et al., Editors. 2001, Garland Publishing. p. 186-220.

19. Costello, P.S., M. Gallagher, and D.A. Cantrell, Sustained and dynamic inositol lipid metabolism inside and outside the immunological synapse. Nat. Immunol., 2002. 3(11): p. 1082-89.

20. Cantrell, D.A., Regulation and function of serine kinase networks in lymphocytes. Curr. Opin. Immunol., 2003. 15: p. 294-98.
21. Haks, M.C., et al., Contributions of the $\mathrm{T}$ cellreceptor-associated CD3g-ITAM to thymocyte selection. J. Exp. Med., 2002. 196(1): p. 1-13.

22. Backstrom, B., et al., Positive selection through a motif in the alphabeta T cell receptor. Science, 1998. 281: p. 835-8.

23. Xia, Z., et al., Opposing effects of ERK and JNK-p38 MAP kinases on apoptosis. Science, 1995. 270: p. 1326-31.

24. Mariathasan, S., et al., Degree of ERK activation influences both positive and negative thymocyte selection. Eur. J. Immunol., 2000. 30: p. 1060-68.

25. Urdahl, K.B., D.M. Pardoll, and M.K. Jenkins, Cyclosporin A inhibits positive selection and delays negative selection in ab TCR transgenic mice. J. Immunol., 1994. 152: p. 2853-59.

26. Kurita-Ochiai, T., et al., Cellular events involved in butyric acid-induced T cell apoptosis. J. Immunol., 2003. 171: p. 3576-84.

27. Roose, J.P., et al., T cell receptor-independent basal signaling via Erk and Abl kinases suppresses RAG gene expression. PLOS Biol., 2003. 1(2): p. 271-87.

28. Ko, M., et al., T-cell receptor signaling inhibits glucocorticoid-induced apoptosis by repressing the SRG3 expression via Ras activation. J. Biol. Chem., 2004. In Press.

29. Mariathasan, S., et al., Duration and strength of extracellular signal-regulated kinase signals are altered during positive versus negative thymocyte selection. J. Immunol., 2001. 167(9): p. 4966-73.

30. Costello, P.S., et al., Ternary complex factor SAP-1 is required for Erk-mediated thymocyte positive selection. Nat. Immunol., 2004. 5(3): p. 289-98.

31. Hogquist, K., RasGRP: the missing link for Ras activation in thymocytes. TREND Immunol., 2001. 22(2): p. 69.

32. Alberola-lla, J. and G. Hernandez-Hoyos, The Ras/MAPK cascade and the control of positive selection. Immunol. Rev., 2003. 191: p. 79-96.

33. Neilson, J.R., et al., Calcineurin B1 Is Essential for Positive but Not Negative Selection during Thymocyte Development. Immunity, 2004. 20(3): p. 255-66.

34. Norment, A.M., et al., Trnasgenic expression of RasGRP1 induces the maturation of double-negative thymocytes and enhances the production of CD8 single-positive thymocytes. J. Immunol., 2003. 170: p. 1141-49.

35. Priatel, J.J., et al., RasGRP1 transduces low-grade TCR signals which are critical for $T$ cell development, homeostasis, and differentiation. Immunity, 2002. 17: p. 617-27.

36. Gong, Q., et al., Disruption of $\mathrm{T}$ cell signaling network and development by Grb2 haploid insufficiecy. Nat. Immunol., 2001. 2(1): p. 29-36.

37. Rincon, M., et al., The JNK pathway regulates the In vivo deletion of immature CD4+CD8+ thymocytes. J. Exp. Med., 1998. 188(10): p. 1817-30. 
38. Sabapathy, K., et al., c-Jun NH2-terminal kinase (JNK)1 and JNK2 have similar and stage-dependent roles in regulating $\mathrm{T}$ cell apoptosis and proliferation. J. Exp. Med., 2001. 193(3): p. 317-28.

39. Sugawara, T., et al., Differential roles of ERK and p38 MAP kinase pathways in positive and negative selection of T lymphocytes. Immunity, 1998. 9(4): p. 565-74.

40. Kikuchi, K., et al., Suppression of thymic development by the dominant-negative form of Gads. Int. Immunol., 2001. 13(6): p. 777-83.

41. Yoder, J., et al., Requirement for the SLP-76 adaptor GADS in T cell development. Science, 2001. 291: p. 1987-91.

42. Clements, J.L., Known and potential functions for the SLP-76 adapter protein in regulating T-cell activation and development. Immunol. Rev., 2003. 191: p. 21119.

43. Thien, C.B.F., D.D.L. Bowtell, and W.Y. Langdon, Perturbed regulation of ZAP-70 and sustained tyrosine phosphorylation of LAT and SLP-76 in cCbl-deficient thymocytes. J. Immunol., 1999. 162: p. 7133-39.

44. Kumar, L., et al., Differential role of SLP-76 domains in $\mathrm{T}$ cell development and function. PNAS, 2002. 99(2): p. 884-889.

45. Tybulewicz, V.L.J., et al., Vav1: a key signal transducer downstream of the TCR. Immunol. Rev., 2003. 192: p. 42-52.

46. Reynolds, L.F., et al., Vav1 transduces T cell receptor signals to the activation of the Ras/ERK pathway via LAT, Sos and RasGRP1. J. Biol. Chem., 2004. 279(18): p. 18239-18246.

47. Ko, M., et al., E2A/HEB and Id3 proteins control the sensitivity to glucocorticoid-induced apoptosis in thymocytes by regulating the SRG3 Expression. J. Biol. Chem., 2004. 279(21): p. 21916-21923.

48. Engel, I. and C. Murre, E2A proteins enforce a proliferation checkpoint in developing thymocytes. EMBO J., 2004. 23: p. 202-11. 